\title{
Fractional Quantum Hall Effect in a Relativistic Field Theory
}

\author{
David B. Kaplan $\oplus^{1, *}$ and Srimoyee Sen $\oplus^{2, \dagger}$ \\ ${ }^{1}$ Institute for Nuclear Theory, Box 351550, University of Washington, Seattle, Washington 98195-1550, USA \\ ${ }^{2}$ Department of Physics and Astronomy, Iowa State University, Ames, Iowa 50011-3160, USA
}

(Received 6 December 2019; revised manuscript received 6 February 2020; accepted 16 March 2020; published 31 March 2020)

\begin{abstract}
We construct a class of $2+1$ dimensional relativistic quantum field theories which exhibit the fractional quantum Hall effect in the infrared, both in the continuum and on the lattice. The UV completion consists of a perturbative $U(1) \times U(1)$ gauge theory with integer-charged fields, while the low energy spectrum consists of nontrivial topological phases supporting fractional currents, bulk anyonic excitations, and exotic phenomena such as a fractional quantum spin Hall effect. We show explicitly how fractionally charged chiral edge states emerge in the IR.
\end{abstract}

DOI: 10.1103/PhysRevLett.124.131601

Introduction.-Topological materials have been discussed in the context of lattice quantum field theories since the early 1990s. In particular, the theory of domain wall fermions (DWF) [1]—designed to optimally realize chiral symmetry on a lattice within the constraints of the NielsenNinomiya theorem [2] - is an example of a topological insulator, a gapped symmetry protected topological phase of matter [3]. In the DWF theory, Chern-Simons (CS) currents in the gapped bulk play the role of quantized Hall currents [4], while the chiral fermions bound to the surfaces [5] are equivalent to the edge states found in the integer quantum Hall system, albeit generalized to non-Abelian gauge theories. A field theoretic generalization of the TKNN calculation [6] was given in Ref. [7], where it was shown that the quantization of the CS operator coefficient (responsible for the quantization of the bulk current) had a topological origin, and that transitions between these topological phases were responsible for abrupt changes seen in the spectrum of chiral edge states [8]. In particular, the Feynman diagram calculation of the CS coefficient in $d+1$ spacetime dimensions was shown to compute the winding number of the map from momentum space to the sphere $S^{d+1}$, explaining its quantization and insensitivity to parameters in the Lagrangian. Furthermore, the constructions of anomaly-free representations for chiral edge states given in Refs. [1,9] (for the purpose of regulating chiral gauge theories) have vanishing gauged but nonzero quantized chiral flavor currents in the bulk, and are realizations of the quantum spin Hall effect. (The simplest anomaly-free theory consists of a Dirac fermion at each edge, as in the

Published by the American Physical Society under the terms of the Creative Commons Attribution 4.0 International license. Further distribution of this work must maintain attribution to the author(s) and the published article's title, journal citation, and DOI. Funded by SCOAP ${ }^{3}$. examples of the SQHE seen experimentally [10,11], although less trivial examples of anomaly cancellation have been studied numerically [9].) In yet another correspondence between relativistic and condensed matter topological materials, Majorana fermion edge states were introduced in the relativistic context [12] and used for the numerical simulation of gauginos in supersymmetric gauge theory [13], analogous to the Majorana modes of condensed matter systems [14].

Despite this long list of topological phases found in condensed matter that also play a role in relativistic quantum field theory, notably missing to date is the fractional quantum Hall effect (FQHE). In this Letter we provide an example of such a theory, by which we mean a perturbative, UV-complete, $2+1$ dimensional, Lorentzinvariant gauge theory of integer-charged matter fields, where the low energy theory at zero chemical potential is characterized by topological phases with fractionally charged chiral edge modes and a fractional coefficient for the Chern-Simons operator in the bulk. Our construction is motivated by existing effective field theory descriptions of IR phenomena in condensed matter systems (see, for example, Refs. $[15,16])$; however, a perturbative UV completion is typically impossible in most physical condensed matter systems due to the high degeneracy of states, and one must simply guess at the appropriate low energy effective theory. In contrast, our relativistic construction allows one to compute in detail how the collective IR behavior arises from short distance physics. The framework provides examples of other exotic phenomena, such as a fractional quantum spin Hall effect, and we are able to demonstrate explicitly how the spectrum of fractionally charged chiral edge modes emerges.

Our theory is a $U(1) \times U(1)$ gauge theory in $2+1$ dimensions, with gauge bosons $A_{\alpha}$ (the "photon") and $Z_{\alpha}$, and three types of fermions $\psi, \chi$, and $\omega$ with charge assignments as shown in Table I. For simplicity we take all 
TABLE I. Dirac fermions labeled by $U(1)_{A} \times U(1)_{Z}$ charge assignments $\left\{q_{A}, q_{Z}\right\}$.

\begin{tabular}{lccc}
\hline \hline & $n_{\text {flavor }}$ & $q_{A}$ & $q_{Z}$ \\
\hline$\psi$ & $n_{\psi}$ & 1 & 0 \\
$\chi$ & $n_{\chi}$ & 0 & 1 \\
$\omega$ & $n_{\omega}$ & 1 & 1 \\
\hline \hline
\end{tabular}

fermion masses to have the same magnitude, $\left|m_{i}\right|=M$, where $M$ is some positive mass scale. Each fermion is assigned an integer flavor number $n_{\psi}, n_{\chi}$, and $n_{\omega}$ respectively, where $\left|n_{i}\right|$ denotes the number of degenerate flavors of fermion type $i$, while the sign of $n_{i}$ equals the sign of the fermion mass, $n_{i} /\left|n_{i}\right| \equiv m_{i} /\left|m_{i}\right|$. The Lagrangian then consists of the usual Dirac terms for the fermions with covariant derivative $D_{\alpha}=\partial_{\alpha}+i q_{A} A_{\alpha}+i q_{Z} Z_{\alpha}$, as well as Maxwell terms for the gauge bosons, $-\left(1 / 4 e^{2}\right) F_{\alpha \beta} F^{\alpha \beta}$, $-\left(1 / 4 g^{2}\right) Z_{\alpha \beta} Z^{\alpha \beta}$, where $Z_{\alpha \beta}$ is the field strength for the $Z_{\alpha}$ gauge boson and $g$ is its coupling constant. The $q_{A}$ and $q_{Z}$ charges we choose for the fermions are given in Table I.

The topological phase structure of the theory depends on momentum space being compact, and so we consider two different regularizations in this Letter with quite different topological phases: we first examine in detail Pauli-Villars field regularization in the continuum, and then later briefly consider a lattice regularization with Wilson fermions, adapting results from previous papers. The former compactifies momentum space to a sphere while the latter to a torus (the Brillouin zone). For the former we take one PauliVillars field of mass $\Lambda$ for each fermion in the theory with mass $M$, with the same charge assignment but opposite statistics and opposite sign mass, $\Lambda /|\Lambda|=-M /|M|$. The opposite statistics is what regulates the UV behavior of fermion loops, while having a relative sign between the mass of each fermion and its regulator allows the theory to be in a nontrivial topological phase, as we will discuss below. With our normalization of the gauge fields, and with $\hbar=c=1$, the theory has four different mass scales: $\Lambda, M, g^{2}$, and $e^{2}$, which we choose to obey the following inequalities,

$$
|\Lambda| \gg|M| \gg g^{2} \gg e^{2},
$$

where the hierarchies are considered to be much bigger than the numbers of flavors, $\left|n_{\psi, \chi, \omega}\right|$.

We now construct the effective low energy theory for this system. Given our hierarchy of scales in Eq. (1), we first integrate out the massive fermions and their regulator fields. The resultant theory is gapped, with the exception of massless chiral edge states if the system has a boundary. The next heaviest state is the $Z$ boson, and so we next integrate out that field, yielding the effective theory for just the photon and the edge states.

First we consider the theory without a boundary, postponing the discussion of edge states. The most relevant gauge invariant operators that can be generated on integrating out the massive fermions are CS operators involving the two gauge fields. In particular we obtain the following contribution to the effective Lagrangian (Our metric convention is $\eta^{\alpha \beta}=\operatorname{diag}\{1,-1,-1\}$ with $\epsilon_{012}=1$ and Dirac matrices satisfying $\left\{\gamma^{\alpha}, \gamma^{\beta}\right\}=2 \eta^{\alpha \beta}$.),

$$
\begin{aligned}
\mathcal{L}_{\mathrm{CS}}= & \frac{\epsilon^{\alpha \beta \gamma}}{4 \pi}\left[n_{\psi} A_{\alpha} \partial_{\beta} A_{\gamma}+n_{\chi} Z_{\alpha} \partial_{\beta} Z_{\gamma}\right. \\
& \left.+n_{\omega}\left(A_{\alpha}+Z_{\alpha}\right) \partial_{\beta}\left(A_{\gamma}+Z_{\gamma}\right)\right]+\cdots,
\end{aligned}
$$

where the ellipses refers to higher derivative operators, including the Maxwell terms. These $P$ and $T$ violating CS operators are proportional to the signs of the fermion and Pauli-Villars masses, which are odd under $P$ and $T$; the opposite sign mass for the Pauli-Villars field, in conjunction with its opposite statistics, ensures that its contribution adds to rather than cancel with the fermion contribution for each fermion. At this point it should be no surprise then that the theory will exhibit the FQHE, since Eq. (2) is an example of the effective description for the FQHE in condensed matter systems [17].

With the Chern-Simons operators being linear in derivatives and the Maxwell terms quadratic, the gauge boson propagators develop gauge-invariant poles at nonzero mass [18]. With $g^{2} \gg e^{2}$, the $Z$ boson is heaviest with mass

$$
M_{Z}=g^{2}\left(n_{\chi}+n_{\omega}\right) /(2 \pi)+O\left(e^{2} / g^{2}\right),
$$

and so we integrate it out of the theory to create the effective theory for the photon. Since we are looking for a theory with momenta $k$ satisfying $M_{Z} \gg k>M_{\gamma}$, we can take $g \rightarrow \infty$ at this point in our calculations, ignoring the irrelevant Maxwell term for the $Z$. Since the theory is gauge invariant, we need to introduce a gauge fixing term, however, $(1 / 2 \xi)\left(Z^{\alpha} \partial_{\alpha} \partial_{\beta} Z^{\beta}\right)$. The $Z$-dependent part of the Lagrangian may then be written as

$$
\begin{aligned}
\mathcal{L}_{Z}= & \frac{1}{2}\left[\left(Z-\frac{n_{\omega}}{2 \pi} A \Delta \mathcal{Q}^{-1}\right) \mathcal{Q}\left(Z-\frac{n_{\omega}}{2 \pi} \mathcal{Q}^{-1} \Delta A\right)\right. \\
& \left.-\left(\frac{n_{\omega}}{2 \pi}\right)^{2} A \Delta \mathcal{Q}^{-1} \Delta A\right],
\end{aligned}
$$

with definitions

$$
\begin{aligned}
\mathcal{Q} & =-\frac{\left(n_{\chi}+n_{\omega}\right)}{2 \pi} \Delta+\frac{\Xi}{\xi}, \\
\Delta^{\alpha \beta} & =\epsilon^{\alpha \beta \gamma} \partial_{\gamma}, \quad \Xi^{\alpha \beta}=\partial^{\alpha} \partial^{\beta} .
\end{aligned}
$$

The propagator is then computed to be

$$
\mathcal{Q}^{-1}=\frac{2 \pi}{\left(n_{\chi}+n_{\omega}\right)} \frac{\Delta}{\partial^{2}}+\frac{\xi \Xi}{\left(\partial^{2}\right)^{2}},
$$


TABLE II. Properties of the low energy spectrum for various values of the UV flavor numbers $\left\{n_{\psi}, n_{\chi}, n_{\omega}\right\}$. The quantity $\nu$ from Eq. (7) is the analogue of the filling fraction in condensed matter systems while $\left\{q_{\psi}^{\prime}, q_{\chi}^{\prime}, q_{\omega}^{\prime}\right\}$ are the charges of the massless chiral edge states; $q_{\phi}^{\prime}$ and $\alpha_{\phi}$ are the charge and statistics of bulk excitations arising from a boson field $\phi$ in the UV with $U(1) \times U(1)$ charges $q_{Z}=1, q_{A}=0$.

\begin{tabular}{lcccr}
\hline \hline$\left\{n_{\psi}, n_{\chi}, n_{\omega}\right\}$ & $\nu$ & $\left\{q_{\psi}^{\prime}, q_{\chi}^{\prime}, q_{\omega}^{\prime}\right\}$ & $q_{\phi}^{\prime}$ & $\alpha_{\phi}$ \\
\hline$\{1,-1,-2\}$ & $1 / 3$ & $\{1,-2 / 3,1 / 3\}$ & $-2 / 3$ & $4 / 3$ \\
$\{0,1,1\}$ & $1 / 2$ & $\{1,-1 / 2,1 / 2\}$ & $-1 / 2$ & $1 / 2$ \\
$\{0,1,2\}$ & $2 / 3$ & $\{1,-2 / 3,1 / 3\}$ & $-2 / 3$ & $2 / 3$ \\
$\{2,1,1\}$ & $5 / 2$ & $\{1,-1 / 2,1 / 2\}$ & $-1 / 2$ & $1 / 10$ \\
\hline \hline
\end{tabular}

from which follows $\Delta \mathcal{Q}^{-1} \Delta=-2 \pi \Delta /\left(n_{\chi}+n_{\omega}\right)$. Therefore, after integrating out the $Z$, we are left with the low energy effective theory for the photon,

$$
\begin{aligned}
\mathcal{L}_{\mathrm{EFT}} & =\frac{1}{4 e^{2}} F_{\alpha \beta} F^{\alpha \beta}+\nu \frac{\epsilon^{\alpha \beta \gamma}}{4 \pi} A_{\alpha} \partial_{\beta} A_{\gamma}+\cdots, \\
\nu & =\left(n_{\psi}+\frac{n_{\chi} n_{\omega}}{n_{\chi}+n_{\omega}}\right),
\end{aligned}
$$

where the ellipses represent irrelevant higher derivative operators. The Hall current for this system with conventional normalization of the gauge field is then given by

$$
J^{\alpha}=\nu \frac{e^{2}}{4 \pi} \epsilon^{\alpha \beta \gamma} F_{\beta \gamma}
$$

implying a fractional Hall conductivity $\sigma_{x y}=\nu e^{2} / h$. In general our expression for $\nu$ takes noninteger values, as displayed for several examples in Table II. While values of $\nu$ from the Laughlin and Jain series can be obtained by suitable choice of flavor numbers, these series play no special role in the relativistic theory.

Fractionally charged chiral surface modes and bulk excitations. - When the system has a boundary, gauge invariance requires that there must be a boundary theory whose $U(1)$ anomaly cancels the divergence of the bulk Chern-Simons current in Eq. (8), the Callan-Harvey effect [4]. Indeed, if one turns off the gauge interactions, one can show that a chiral edge state exists for each flavor of fermion with chirality proportional to the sign of the bulk fermion mass [5]; however, from their charge assignments in Table I it appears that they can only carry integer charge under the $U(1)_{A}$ gauge group and therefore cannot have the correct chiral anomaly to match fractional CS current in the bulk. To resolve this puzzle we consider the theory on the half-space $x^{2} \geq 0$ with boundary condition for both the fermions and Pauli-Villars fields of type $i$

$\left.P_{i}^{+} \Psi_{i}\right|_{x^{2}=0}=\left.P_{i}^{-} \Psi_{i}\right|_{x^{2}=\infty}=0, \quad P_{i}^{ \pm}=\frac{\left[1 \pm \epsilon\left(n_{i}\right) \Gamma\right]}{2}$, where $n_{i}$ is the flavor number of that fermion (recall that for each fermion the sign of $n$ reflects the underlying sign chosen for its mass), and $\Gamma=i \gamma^{2}$ is the Hermitian chiral matrix with eigenvalues \pm 1 . Massless chiral edge states correspond to solutions to $\left(i \gamma^{2} \partial_{2}-m_{i}\right) \Psi_{i}=0$ consistent with the boundary condition Eq. (9). Such states exist for each fermion with chirality given by $-\epsilon\left(n_{i}\right)$. The PauliVillars fields of type $i$ have opposite sign mass (but the same $n_{i}$ ) and the boundary condition forbids a zero mode solution for them.

To compute the effective theory for the half-space we must include the massless edge modes, adding to the UV action $\int d^{3} x\left(A_{\mu} \mathcal{J}_{A}^{\mu}+Z_{\mu} \mathcal{J}_{Z}^{\mu}\right)$, where the currents have the form dictated by the charges $q_{A, Z}$ given in Table I

$$
\begin{aligned}
\mathcal{J}_{A}^{\mu} & =\left(\mathcal{J}_{\psi}^{\mu}+\mathcal{J}_{\omega}^{\mu}\right), & \mathcal{J}_{Z}^{\mu} & =\left(\mathcal{J}_{\chi}^{\mu}+\mathcal{J}_{\omega}^{\mu}\right), \\
\mathcal{J}_{i}^{\mu} & =\delta\left(x^{2}\right) \bar{\Psi}_{i} \gamma^{\mu} P_{i}^{-} \Psi_{i}, & i & =\psi, \chi, \omega,
\end{aligned}
$$

where $\mu=\{0,1\}$ designates the $d=1+1$ coordinates on the mass defect, and the $\Psi_{i}$ only include fermions, not Pauli-Villars fields. Integrating out the $Z$ boson therefore induces couplings between $\mathcal{J}_{Z}^{\mu}$ and the photon, and we must add to the effective theory in Eq. (7) the edge mode interactions

$$
\begin{aligned}
\mathcal{L}_{\mathrm{EFT}}^{\mathrm{zm}}= & \frac{n_{\omega}}{4 \pi}\left[A^{\alpha}\left(\Delta \mathcal{Q}^{-1}\right)_{\alpha \beta} \mathcal{J}_{Z}^{\beta}+\mathcal{J}_{Z}^{\alpha}\left(\mathcal{Q}^{-1} \Delta\right)_{\alpha \beta} A^{\beta}\right] \\
& +A_{\mu} \mathcal{J}_{A}^{\mu}, .
\end{aligned}
$$

In the above equation indices $\alpha, \beta$ take values in $2+1$ dimensions, but the bulk component of the zero mode current vanishes, $\mathcal{J}_{Z}^{2}=0$. If we combine the contributions to the photon current from the first two terms above with the bulk contribution from the last term in Eq. (4), we find that the photon current induced by integrating out the $Z$ is given by

$$
J_{\text {induced }}^{\alpha}=\frac{n_{\omega}}{2 \pi}\left(\Delta \mathcal{Q}^{-1}\left[\mathcal{J}_{Z}-\frac{n_{\omega}}{2 \pi} \Delta A\right]\right)^{\alpha} .
$$

The quantity in parentheses is the conserved current identified by Callan and Harvey [4], the divergence of the bulk current at the boundary being canceled by the anomaly of the edge state current. (The cancellation requires one use the covariant anomaly for the edge states instead of the consistent anomaly, which is half as large; the discrepancy is resolved by the generation of additional gauge field terms at the surface [19].) The operator acting on this current is

$$
\left(\Delta \mathcal{Q}^{-1}\right)^{\alpha \beta}=-\frac{2 \pi}{n_{\chi}+n_{\omega}}\left(\eta^{\alpha \beta}-\frac{\partial^{\alpha} \partial^{\beta}}{\partial^{2}}\right),
$$

which is recognized as being proportional to the projection operator onto physical states. Without altering the physics 
we can make the interaction local by adding a similar term involving the projection operator onto unphysical longitudinal states, since the added interaction is proportional to the divergence of the conserved current, allowing us to replace

$$
\left(\Delta \mathcal{Q}^{-1}\right)^{\alpha \beta} \rightarrow-\frac{2 \pi}{n_{\chi}+n_{\omega}} \eta^{\alpha \beta} .
$$

Adding the resultant induced current to the direct zero mode contribution $\mathcal{J}_{A}^{\mu}$ in Eq. (11) yields the total interaction of the photon with the edge states

$$
\mathcal{J}_{A, \mathrm{EFT}}^{\mu}=\left(q_{\psi}^{\prime} \mathcal{J}_{\psi}^{\mu}+q_{\chi}^{\prime} \mathcal{J}_{\chi}^{\mu}+q_{\omega}^{\prime} \mathcal{J}_{\omega}^{\mu}\right)
$$

with

$$
q_{\psi}^{\prime}=1, \quad q_{\chi}^{\prime}=-\frac{n_{\omega}}{n_{\chi}+n_{\omega}}, \quad q_{\omega}^{\prime}=1-\frac{n_{\omega}}{n_{\chi}+n_{\omega}},
$$

taking into account the $\left(n_{\omega} / 4 \pi\right)$ prefactor in Eq. (11). We see therefore that in the low energy theory, the chiral $\chi$ and $\omega$ edge states carry fractional charge. For example, our $\nu=1 / 3$ example from Table II yields the fractional charges $q_{\chi}^{\prime}=-2 / 3$ and $q_{\omega}^{\prime}=1 / 3$ for the chiral $\chi$ and $\omega$ edge states, respectively.

For our effective theory to be gauge invariant, the $d=1+1$ anomaly at the edge of the sample with these fractionally charged edge modes must correctly cancel the boundary divergence of the Hall current Eq. (8). Indeed this is the case since

$$
\sum_{i=\psi, \chi, \omega} n_{i}\left(q_{i}^{\prime}\right)^{2}=\left(n_{\psi}+\frac{n_{\chi} n_{\omega}}{n_{\chi}+n_{\omega}}\right)=\nu .
$$

Bulk excitations in the IR can be studied by adding additional light fields to the theory. Consider, for example, a bosonic field $\phi$ of mass $m_{\phi}$ with $m_{\phi}<M_{Z}$ and charges $q_{A}=0, q_{Z}=1$. After integrating out the $Z$ field, $\phi$ couples to the photon with induced fractional charge given by

$$
q_{\phi}{ }^{\prime}=q_{\chi}^{\prime}=-\frac{n_{\omega}}{n_{\chi}+n_{\omega}} .
$$

A magnetic flux $2 \pi q_{\phi}^{\prime} / \nu$ attaches to the particle in the IR, and so in the usual way the resulting Aharanov-Bohm phase modifies the boson statistics so that the two-boson state satisfies $\left|\psi_{1} \psi_{2}\right\rangle=e^{i \alpha_{\phi}}\left|\psi_{2} \psi_{1}\right\rangle$ with

$\alpha_{\phi}=\frac{\left(q_{\phi}^{\prime}\right)^{2}}{\nu}=\frac{n_{\omega}^{2}}{\left(n_{\chi}+n_{\omega}\right)\left(n_{\chi} n_{\psi}+n_{\omega} n_{\psi}+n_{\chi} n_{\omega}\right)}$.

Examples of the fractional coupling and statistics for these excitations are in Table II. Different initial $q_{A, Z}$ charges will lead to bulk excitations with different behavior in the IR theory.

Fractional quantum spin Hall effect.-The quantum spin Hall effect (QSHE) occurs when the current in the bulk transports global quantum numbers but not gauged charges, in contrast with the classical Hall current. In the language of quantum field theory, this means that the edge states form an anomaly-free representation of the gauge group, while various flavor symmetries can have gauge anomalies. This effect was analyzed in Ref. [1], where it was suggested that by physically separating topologically protected edge states in gauge anomaly free representations from their conjugate counterparts at the opposite edge, one could turn the problem of finding a nonperturbative regulator for chiral gauge theories into finding the appropriate phase of a lattice theory with spatially dependent interactions. (This idea is still being pursued in various forms [20-25].). By construction, such materials only have CS (Hall) currents which carry flavor quantum numbers in the bulk. The simplest example is a material with a massless Dirac fermion at each edge, such as seen in condensed matter systems [26], but less trivial examples of anomaly cancellation have been investigated numerically, such as the 3-4-5 model [9] which has a chiral representation on the boundary.

It is evident that a fractional quantum spin Hall effect is possible, where the Hall currents only transport fractional flavor charges. In order to not have transport of electric charge we must have the coefficient $\nu$ of the Hall current in Eq. (8) vanish, namely,

$$
\nu=\left(n_{\psi}+\frac{n_{\chi} n_{\omega}}{n_{\chi}+n_{\omega}}\right)=0 .
$$

We can then consider the transport of any linear combination of the many $U(1)$ flavor currents in the model, normalized so that the fermions in the UV theory carry integer charge, and compute its Hall current. As a specific example, consider the flavor current $j^{\alpha}$ of a single one of the $\left|n_{\chi}\right| \chi$ fermions, e.g., $\chi_{1}$. We can introduce a source $f_{\alpha}$ in the UV theory coupled to the current $\bar{\chi}_{1} \gamma^{\mu} \chi_{1}$ and compute the effective theory with all fields integrated out except for $f_{\alpha}, A_{\alpha}$ and the chiral edge states. The flavor current in the IR can be obtained by differentiating this action with respect to $f_{\alpha}$, then setting $f_{\alpha}=0$. A nontrivial result for the Hall conductivity of our flavor current will be obtained when the effective theory has an induced Chern-Simons coupling between $f_{\alpha}$ and $A_{\alpha}$. This is the case with our particular example, and we find that the Hall conductivity for this flavor is

$$
\sigma_{x y}^{\left(\chi_{1}\right)}=-2 \frac{n_{\omega}}{n_{\omega}+n_{\chi}} \frac{e^{2}}{h}=2 \frac{n_{\psi}}{n_{\chi}} \frac{e^{2}}{h} .
$$

For example, if we set $n_{\psi}=2, n_{\chi}=-6$ and $n_{\omega}=-3$ we solve the anomaly cancellation Eq. (20, while the Hall 
conductivity for the $\chi_{1}$ number given by Eq. (21) is $\sigma_{x y}^{\left(\chi_{1}\right)}=-\frac{2}{3}\left(e^{2} / h\right)$, and this fractional Hall conductivity will be accompanied by massless edge modes carrying a fractional $\chi_{1}$-flavor number as well, to ensure flavor current conservation. (FQSHE has been previously discussed for theories possessing time-reversal symmetry in Ref. [27]. None of the models considered here are $T$ invariant, which would require all fermions to come in \pm sign mass pairs.)

Topological phases.- Ref. [7] explained the quantization of the CS coefficient as being topological in origin, equal to the winding number of the map provided by the fermion dispersion relation from momentum space to the Dirac sphere. This winding number only has meaning in the IR and depends on the fermions being massive (gapped) in the bulk. Topological phase transitions can occur at points or surfaces in parameter space where the theory becomes gapless. It was the observation of discontinuous jumps in the spectrum of chiral edge states in lattice quantum field theory at particular parameter values that motivated that work.

In the example we have analyzed here, each fermion of mass $M$ and its accompanying Pauli-Villars regulator of mass $\Lambda$ combined contribute a factor to the coefficient of the CS operator proportional to $(M /|M|-\Lambda /|\Lambda|)= \pm 2$, given our choice that $\Lambda /|\Lambda|=-M /|M|$. Evidently if we had chosen the Pauli-Villars mass to have the same sign as the fermion, the two contributions would cancel, the magnitude of the fractional Hall current would change, and the charges of the chiral edge modes would be different. Therefore a topological phase transition can be seen by varying the fermion mass continuously from $M \rightarrow-M$, where the transition occurs at $M=0$ and the CS operator coefficient changes by \pm 2 . However, our boundary condition (9) would also change discontinuously which makes this example difficult to analyze. So instead of a half space, consider the full space, but where the fermion mass $M$ is positive for one sign of $x^{2}$ and negative for the other, so that the chiral edge modes live on the "domain wall" at $x^{2}=0$. The Pauli-Villars mass is taken to be constant everywhere so that there are no massless edge states with negative norm. Now we see that the sum of fermion and Pauli-Villars contributions add on one side of the circle and cancel on the other, so that the transformation $M \rightarrow-M$ has the effect of a parity transformation, moving the nonzero CS current from one side of $x^{2}=0$ to the other and flipping the chirality of the edge modes, but not rendering the topology trivial.

Instead of Pauli-Villars regularization we could employ a lattice regularization with Wilson fermions. In this case one finds a much richer topological phase diagram, where one can vary the ratio of the fermion mass to Wilson coefficient $M / r$ for each fermion (The Wilson parameter $r$ refers to the coefficient of the $\bar{\psi} D^{2} \psi$ operator, where $D^{2}$ is the gauge covariant lattice Laplacian.), toggling the number and chirality of edge states for each flavor through the
Pascal triangle numbers $0,1,2,1,0$ with alternating chirality; see, for example, Refs. [7,28] for a discussion and phase diagram. Thus, for a single Wilson fermion field with $q_{A}=0$ and $q_{Z}=1$ we get a contribution to $n_{\chi}$ equal to one of the values $\{0, \pm 1, \pm 3\}$ depending on the choice of $M / r$ for that field. Since such choices can be made individually for each fermion field in the UV, we see that the lattice theory will have a large number of possible topological phases for any particular set of fermions.

Discussion.-By adopting existing effective field theory descriptions of the FQHE in condensed matter systems we have explicitly constructed relativistic UV completions that exhibit the same phenomenon of charge fractionalization. These theories have only integer charged fermions and gauge fields in the UV, and they exhibit a topological phase structure which (as should be expected) is dependent on the specifics of the UV regularization used. Such theories are perturbative and the emergence of topological IR physics, such as fractionalization of the charges of the chiral edge fermions, can be explicitly computed. Similarly it is easy to construct UV theories that exhibit a fractional spin quantum Hall effect. There are many ways one can extend this approach, such as by extending the gauge group to include more Abelian or non-Abelian gauge fields-for example, starting with the effective field theories in Ref. [29]—and to incorporate spontaneous symmetry breaking or other dimensions. (There have been several recent papers investigating the topological phase diagram of $\mathrm{U}(1)$ gauge theories on the lattice in $1+1$ dimensions [30-33].) It is hoped that in pursuing this program it may be possible to further our understanding of topological phenomena and how they can arise, although it is not clear whether this could be relevant for relativistic theories in $3+1$ dimensions.

This research was supported in part by DOE Grant No. DE-FG02-00ER41132 and Iowa State University startup funds. We thank Andreas Karch for useful conversations.

*dbkaplan@uw.edu 'srimoyee08@gmail.com

[1] D. B. Kaplan, Phys. Lett. B 288, 342 (1992).

[2] H. B. Nielsen and M. Ninomiya, Nucl. Phys. B185, 20 (1981).

[3] X.-G. Wen, Phys. Rev. B 40, 7387 (1989).

[4] J. Callan, G. Curtis, and J. A. Harvey, Nucl. Phys. B250, 427 (1985).

[5] R. Jackiw and C. Rebbi, Phys. Rev. D 13, 3398 (1976).

[6] D. J. Thouless, M. Kohmoto, M. P. Nightingale, and M. den Nijs, Phys. Rev. Lett. 49, 405 (1982).

[7] M. F. L. Golterman, K. Jansen, and D. B. Kaplan, Phys. Lett. B 301, 219 (1993).

[8] K. Jansen and M. Schmaltz, Phys. Lett. B 296, 374 (1992).

[9] K. Jansen, Phys. Lett. B 288, 348 (1992).

[10] M. König, S. Wiedmann, C. Brüne, A. Roth, H. Buhmann, L. W. Molenkamp, X.-L. Qi, and S.-C. Zhang, Science 318, 766 (2007). 
[11] D. Hsieh, D. Qian, L. Wray, Y. Xia, Y. S. Hor, R. J. Cava, and M. Z. Hasan, Nature (London) 452, 970 (2008).

[12] D. B. Kaplan and M. Schmaltz, Chin. J. Phys. 38, 543 (2000).

[13] M. G. Endres, Phys. Rev. D 79, 094503 (2009).

[14] A. Y. Kitaev, Phys. Usp. 44, 131 (2001).

[15] E. Witten, Riv. Nuovo Cimento 39, 313 (2016).

[16] D. Tong, arXiv:1606.06687.

[17] X.-G. Wen and A. Zee, Phys. Rev. B 46, 2290 (1992).

[18] S. Deser, R. Jackiw, and S. Templeton, Ann. Phys. (N.Y.) 140, 372 (1982); 281, 409 (2000).

[19] S. G. Naculich, Nucl. Phys. B296, 837 (1988).

[20] X.-G. Wen, Chin. Phys. Lett. 30, 111101 (2013).

[21] J. Wang and X.-G. Wen, arXiv:1307.7480.

[22] M. DeMarco and X.-G. Wen, arXiv:1706.04648.

[23] J. Wang and X.-G. Wen, Phys. Rev. D 99, 111501(R) (2019).

[24] D. M. Grabowska and D. B. Kaplan, Phys. Rev. Lett. 116, 211602 (2016).
[25] D. M. Grabowska and D. B. Kaplan, Phys. Rev. D 94, 114504 (2016).

[26] M. Z. Hasan and C. L. Kane, Rev. Mod. Phys. 82, 3045 (2010).

[27] M. Levin and A. Stern, Phys. Rev. B 86, 115131 (2012).

[28] D. B. Kaplan, Modern Perspectives in Lattice QCD: Quantum Field Theory and High Performance Computing, in Proceedings, International School, 93rd Session, Les Houches, France, 2009 (2009), pp. 223-272.

[29] N. Seiberg and E. Witten, Prog. Theor. Exp. Phys. 2016, 12C101 (2016).

[30] G. Magnifico, D. Vodola, E. Ercolessi, S. P. Kumar, M. Müller, and A. Bermudez, Phys. Rev. D 99, 014503 (2019).

[31] A. Bermudez, E. Tirrito, M. Rizzi, M. Lewenstein, and S. Hands, Ann. Phys. (Amsterdam) 399, 149 (2018).

[32] E. Tirrito, M. Rizzi, G. Sierra, M. Lewenstein, and A. Bermudez, Phys. Rev. B 99, 125106 (2019).

[33] G. Magnifico, D. Vodola, E. Ercolessi, S. P. Kumar, M. Müller, and A. Bermudez, Phys. Rev. B 100, 115152 (2019). 